Research Article

\title{
Stochastic Single Machine JIT Scheduling with Geometric Processing Times and Due Dates
}

\author{
Yuncheng Luo \\ School of Mathematics, Shanghai University of Finance and Economics, Shanghai 200433, China \\ Correspondence should be addressed to Yuncheng Luo; luoyuncheng@163.shufe.edu.cn
}

Received 27 September 2020; Revised 19 March 2021; Accepted 26 April 2021; Published 18 May 2021

Academic Editor: Frederic Kratz

Copyright (C) 2021 Yuncheng Luo. This is an open access article distributed under the Creative Commons Attribution License, which permits unrestricted use, distribution, and reproduction in any medium, provided the original work is properly cited.

In this paper, we investigate a static stochastic single machine JIT scheduling problem in which the jobs' processing times are stochastically independent and follow geometric distributions whose mean is provided, due dates are geometrically distributed with a common mean, and both the unit penalty of earliness/tardiness and the fixed penalty of earliness/tardiness are deterministic and different. The objective is to minimize the expected total penalties for quadratic earliness, quadratic tardiness, and early and tardy jobs. We prove that the optimal schedule to minimize this problem is V-shaped with respect to the ratio of mean processing time to unit tardiness penalty under the specific condition. Also, we show a special case and two theorems related to this JIT scheduling problem under specific situations where the optimal solutions exist. Finally, based on the V-shaped characteristic, a dynamic programming algorithm is designed to achieve an optimal V-shaped schedule in pseudopolynomial time.

\section{Introduction}

In just-in-time (JIT) scheduling, the decision maker intends to find a rational scheduling scheme so that all jobs will not be completed either too early or too late. In general, it is assumed that there are job due dates in the JIT scheduling model, and both earliness and tardiness are penalized. This paper tackles a scheduling model involving earliness and tardiness penalties in both quadratic and fixed form, that is, these penalties contain not only the variable charges hinging upon the quadratic earliness/tardiness of the jobs but also the fixed charges generated once the job is not punctually finished no matter how much the earliness/tardiness is. Hence, in order to avoid these penalties as much as possible, it is of great importance for decision maker to allocate the job due dates.

The single machine environment plays a crucial role in scheduling theory. The performance of complex systems such as parallel machines generally depends on the schedule quality of a single bottleneck machine. Also, some results and insights obtained from the research of the single machine problems can be regarded to be valuable for solving the scheduling problems on more complex systems. The scheduling models with a single processor seem to appear frequently in practice. For the deterministic version of the models, researchers have studied many scheduling problems and obtained corresponding optimization results. Unfortunately, a large quantity of scheduling problems we encounter in practical are filled with many uncertain factors, so extending the model to the field of stochastic scheduling is an attempt to make the scheduling theory more practical. However, the research progress related to scheduling problem in the stochastic model has been slow. Hence, the scheduling problems under stochastic environment are still a significant and challenging research direction.

The deterministic models related to single machine JIT scheduling problem have become an extensive research topic discussed since the later 1970s. Sidney [1] was one of the pioneers to study this class of scheduling problem. Kanet [2] proposed a constructive algorithm to find an optimal solution in polynomial time for the scheduling problem, where earliness and tardiness penalties are unit weights. Lann and Mosheiov [3], firstly, investigated the problem with the number of early and tardy jobs related to several cost structures by some heuristic algorithms. Later, there were many scheduling problems with earliness and tardiness 
penalties which have been studied in [4-6] by different algorithms, whilst this type of scheduling problem with controllable processing times was discussed in $[7,8]$, and the notion of job preemption in this type of scheduling problem was also examined in $[9,10]$. The problems of minimizing the quadratic earliness and tardiness have also been considered. In [11-13], researchers, under different assumptions, considered the problem in which all quadratic earliness and tardiness penalties are equal, and this problem with symmetric weighted quadratic penalty function was also studied in $[14,15]$, while the asymmetric weighted version of this problem was investigated in [16-18]. The relevant scheduling problems with quadratic cost function have also been studied in $[19,20]$. For the problems with more than two types of penalties, Lee et al. [21] developed a dynamic programming algorithm to solve two instances of the problem which includes a combination of three types of penalties, namely, earliness, tardiness, and tardy jobs. Shabtay [22] considered a case with the objective function $\sum_{i=1}^{n}\left(f\left(d_{i}\right)+g\left(E_{i}\right)+h\left(T_{i}\right)\right)$, where $f(\cdot), g(\cdot)$, and $h(\cdot)$ are nondecreasing functions. Koulamas [23] presented an algorithm for the $1\left|d_{i}=d\right| \sum_{i=1}^{n}\left(\alpha d+\beta E_{i}+\gamma T_{i}+\delta_{i} V_{i}+\right.$ $\left.\theta_{i} U_{i}\right)$ problem. Baker and Scudder [24] presented an excellent survey for the initial research situations on both linear E-T and quadratic E-T scheduling problems, whilst a brilliant review of scheduling problems with multiple types of penalties was also provided in [25]. In [26, 27], the concept of batch in JIT scheduling problems with different objectives was investigated by several authors.

Although there are most of JIT problems in which processing times and due dates are certain, jobs usually have random processing times and due dates in many practical situations. Surprisingly, there is little literature studied on scheduling problems in stochastic environments. Soroush and Fredendall [28] analyzed the single machine scheduling problem with normally distributed processing times and deterministic due dates to minimize the total expected earliness and tardiness penalties. Baker [29] studied the same case and obtained optimal solutions by designing a branch and bound algorithm. However, this problem was also considered in [30] when both processing times and due dates follow exponential distributions, and they derived the optimal V-shaped schedule by giving a dynamic programming algorithm. Liu and Liu [31] dealt with this type of problem in which release times and processing times are random variables. Cai and Zhou [32] investigated a problem with the three types of penalties, namely, earliness, tardiness, and flow time under normally distributed processing times and identically distributed due dates. Soroush [33] firstly explored the problem whose goal is to minimize the total expected weighted number of early and tardy jobs, where processing times are arbitrarily distributed, due dates are certain and jobs are penalized by fixed earliness weights and tardiness weights. The majority of the corresponding problems with E-T penalties have also been studied in [34-36] under distinct constraint conditions and assumptions. The stochastic version of problems with a relevant quadratic cost function has also been studied by several authors. Mittenthal and Raghavachari [37] analyzed a problem with quadratic earliness and tardiness penalties and stochastic machine breakdowns, whilst processing times are deterministic and due dates are common with certainty, and their objective is to minimize the expected value of the weighted sum of symmetric quadratic earliness and tardiness penalties of all jobs. Cai and Zhou [38] considered a similar case with uncertain uptimes and downtimes, but where both processing times and due dates are exponentially distributed. The problem of stochastically minimizing maximum lateness was analyzed in [39], where processing times and due dates follow exponential distribution. In [40], Soroush and Alqallaf investigated the stochastic problem of finding an optimal sequence that minimizes the total expected costs with weight quadratic tardiness penalties on a single machine, where idle time is not allowed, whilst the extending problem with initial idle time was examined in [41].

In this paper, we, under stochastic scenario, address a static single machine JIT scheduling problem with the objective of minimizing the expected total penalties for quadratic earliness, quadratic tardiness, and early and tardy jobs. In this scheduling problem, it is assumed that processing times follow geometric distributions with distinct parameters and due dates follow geometric distributions with a common parameter. Geometric distribution is a commonly used discrete distribution, where there is only one parameter which represents the probability of success of the event, and the value range of geometric random variable is all positive integers. It is also one of the simplest forms in processing time distributions. Moreover, the jobs are penalized by variable weights depending on quadratic earliness/tardiness and fixed earliness/tardiness weights. These weights are distinct and job-dependent. We show that the optimal solution of this problem has $\mathrm{V}$-shaped characteristic with respect to the ratio of mean processing time to unit tardiness penalty, that is, the schedule will first arrange jobs in nonincreasing order of the ratio of mean processing time to unit tardiness penalty and then arrange jobs in nondecreasing order of the ratio of mean processing time to unit tardiness penalty. To the best of the author's knowledge, no other research, under geometrically distributed processing times and due dates, has studied this objective function which simultaneously incorporated the four types of penalty.

This paper is structured as follows. Section 2 defines and formulates a static single machine stochastic JIT scheduling problem. In Section 3, we obtain the V-shaped property of the optimal schedule for this problem and show a special case and two theorems related to this problem under specific situations where the optimal solutions exist. The designed dynamic programming is presented to obtain an optimal V-shaped schedule in Section 4. Section 5 is the conclusions.

\section{Problem Definition and Formulation}

Consider stochastic JIT scheduling problem that a set $J=$ $\{1,2, \ldots, n\}$ of $n$ jobs are to be scheduled on a single machine. Assume that all jobs prepare for processing at time zero, and job preemption is not allowed that, as soon as a job starts to process on a single machine, it can not be disrupted 
until the process is finished. In addition, it is assumed that the machine which is always available can handle at most one job at a time, no machine breakdown occurs, and the machine inserted idle time are not allowed. In this problem, the processing time $P_{i}$ of each job $i$ is assumed to be independent random variable which follows geometric distribution with parameter $\left(1 / \theta_{i}\right)$. Each job $i$ has a due date $D_{i}$ which has geometric distribution with common parameter $(1 / \delta)$, and $D_{i}$ is independent of $P_{i}$.s

The objective is to determine a schedule $\pi$ that minimizes the expected total penalties for quadratic earliness, quadratic tardiness, and early and tardy jobs which can be formulated as follows:

$$
\operatorname{ETP}(\pi)=E\left[\sum_{i=1}^{n}\left(\alpha_{i} E_{i}^{2}+\beta_{i} T_{i}^{2}+\tau_{i} V_{i}+\varphi_{i} U_{i}\right)\right],
$$

where

$\pi$ determines the order of processing the $n$ jobs is a sequence

$C_{i}(\pi)$ denotes the completion time of job $i$ under $\pi$

$D_{i}$ denotes the due date of job $i$
$E_{i}$ denotes the earliness of job $i$, where $E_{i}=\max$ $\left\{0, D_{i}-C_{i}(\pi)\right\}$

$T_{i}$ denotes the tardiness of job $i$, where $T_{i}=\max$ $\left\{0, C_{i}(\pi)-D_{i}\right\}$

$V_{i}$ denotes the early indictor variable of job $i$, where $V_{i}$ $=1$ if $C_{i}(\pi)<D_{i}$, otherwise, $V_{i}=0$

$U_{i}$ denotes the tardy indictor variable of job $i$, where $U_{i}=1$ if $C_{i}(\pi)>D_{i}$, otherwise, $U_{i}=0$

$\alpha_{i} \geq 0$ is unit earliness penalty of job $i$

$\beta_{i} \geq 0$ is unit tardiness penalty of job $i$

$\tau_{i} \geq 0$ is fixed earliness penalty of job $i$

$\varphi_{i} \geq 0$ is fixed tardiness penalty of job $i$

$E(Y)$ represents the expectation of a random variable $Y$

\section{The V-Shaped Characteristic of the Optimal Schedule}

In this section, our goal is to obtain the structure characteristic of the optimal sequence that minimizes $\operatorname{ETP}(\pi)$, and the objective in (1) can be equivalently expressed into

$$
\begin{aligned}
\operatorname{ETP}(\pi) & =\sum_{i=1}^{n}\left(\tau_{i}-\varphi_{i}\right) \operatorname{Pr}\left(D_{i}>C_{i}\right)+\sum_{i=1}^{n}\left(\alpha_{i}-\beta_{i}\right) E\left[\left(D_{i}-C_{i}\right)^{2} I_{\left\{D_{i}>C_{i}\right\}}\right]+\sum_{i=1}^{n} \beta_{i} E\left[\left(C_{i}-D_{i}\right)^{2}\right]+\sum_{i=1}^{n} \varphi_{i} \\
& =\operatorname{ETP}_{1}(\pi)+\operatorname{ETP}_{2}(\pi)+\operatorname{ETP}_{3}(\pi)+\sum_{i=1}^{n} \varphi_{i},
\end{aligned}
$$

where $I_{A}$ is an indicative function, which takes value 1 when event $A$ occurs; otherwise, it takes 0 , and $\operatorname{ETP}_{i}(\pi)$, $i=1,2,3$, are defined by

$$
\begin{aligned}
& \operatorname{ETP}_{1}(\pi)=\sum_{i=1}^{n}\left(\tau_{i}-\varphi_{i}\right) \operatorname{Pr}\left(D_{i}>C_{i}\right), \\
& \operatorname{ETP}_{2}(\pi)=\sum_{i=1}^{n}\left(\alpha_{i}-\beta_{i}\right) E\left[\left(D_{i}-C_{i}\right)^{2} I_{\left\{D_{i}>C_{i}\right\}}\right] \\
& \operatorname{ETP}_{3}(\pi)=\sum_{i=1}^{n} \beta_{i} E\left[\left(C_{i}-D_{i}\right)^{2}\right] .
\end{aligned}
$$

In order to tackle this scheduling problem, we first present a lemma below.

Lemma 1. If the processing times $\left\{P_{i}, i=1,2, \ldots, n\right\}$ are stochastically independent and geometrically distributed random variables with the mean $\left\{\theta_{i}, i=1,2, \ldots, n\right\}$, the due dates follow geometric distributions with a common mean $\delta$, independent of $\left\{P_{i}\right\}$. Then, given an arbitrary schedule $\pi=\ldots, i, j, \ldots$, let $\pi^{\prime}=\ldots, j, i, \ldots$ denote the schedule that interchanges order of job $i$ and job $j$ in $\pi$. Hence,

$$
\begin{aligned}
& \operatorname{ETP}_{1}(\pi)-\operatorname{ETP}_{1}\left(\pi^{\prime}\right)=\left(\left(\varphi_{j} \theta_{i}-\varphi_{i} \theta_{j}\right)-\left(\tau_{j} \theta_{i}-\tau_{i} \theta_{j}\right)\right) \frac{y_{i} y_{j}}{\delta-1} \prod_{h \in Z^{*}} y_{h}, \\
& \operatorname{ETP}_{2}(\pi)-\operatorname{ETP}_{2}\left(\pi^{\prime}\right)=\left(2 \delta^{2}-\delta\right)\left(\left(\beta_{j} \theta_{i}-\beta_{i} \theta_{j}\right)-\left(\alpha_{j} \theta_{i}-\alpha_{i} \theta_{j}\right)\right) \frac{y_{i} y_{j}}{\delta-1} \prod_{h \in Z^{*}} y_{h}, \\
& \operatorname{ETP}_{3}(\pi)-\operatorname{ETP}_{3}\left(\pi^{\prime}\right)=\left(\beta_{j} \theta_{i}-\beta_{i} \theta_{j}\right)\left(2 \sum_{h \in Z^{*}} \theta_{h}+2 \theta_{i}+2 \theta_{j}-2 \delta-1\right) .
\end{aligned}
$$


Proof. Due to the geometrically distributed of $\left\{P_{i}\right\}$ and $\left\{D_{i}\right\}$, we have $\mathrm{EP}_{i}=\theta_{i}, \quad \operatorname{Var} P_{i}=\sigma_{i}^{2}=\theta_{i}{ }^{2}-\theta_{i}, \quad \mathrm{ED}_{i}=\delta$, and $\mathrm{ED}_{i}^{2}=2 \delta^{2}-\delta$. Therefore, we have the expected completion time of job $i$ under $\pi$ :

$$
\mathrm{EC}_{i}=\sum_{h \in Z_{i}} \mathrm{EP}_{h}=\sum_{h \in Z_{i}} \theta_{h}
$$

where $Z_{i}=Z_{i}(\pi)=\{\ldots, i\}$ is the set of jobs which is to be processed before job $i+1$ in $\pi$. Since $\left\{D_{i}\right\}$ and $\left\{P_{i}\right\}$ are geometrically distributed, which are stochastically independent, we have

$$
\begin{aligned}
\operatorname{Pr}\left(D_{i}>P_{i}\right) & =E\left(\operatorname{Pr}\left(D_{i}>p_{i} \mid P_{i}=p_{i}\right)\right) \\
& =E\left(\sum_{n=p_{i}+1}^{+\infty} \frac{1}{\delta}\left(1-\frac{1}{\delta}\right)^{n-1}\right)=E\left(1-\frac{1}{\delta}\right)^{p_{i}} \\
& =\sum_{n=1}^{+\infty}\left(1-\frac{1}{\delta}\right)^{n} \frac{1}{\theta_{i}}\left(1-\frac{1}{\theta_{i}}\right)^{n-1}=\frac{\delta-1}{\theta_{i}+\delta-1}=y_{i},
\end{aligned}
$$

and

$$
\begin{aligned}
\operatorname{Pr}\left(D_{i}>C_{i}\right) & =E\left(1-\frac{1}{\delta}\right)^{C_{i}}=E\left(1-\frac{1}{\delta}\right)^{\sum_{h \in Z_{i}} P_{h}} \\
& =\prod_{h \in Z_{i}} E\left(1-\frac{1}{\delta}\right)^{P_{h}}=\prod_{h \in Z_{i}} y_{h},
\end{aligned}
$$

where $y_{h}=\left((\delta-1) /\left(\theta_{h}+\delta-1\right)\right)$, which implies

$$
1-y_{i}=\frac{\theta_{i}}{\theta_{i}+\delta-1}=\frac{\theta_{i} y_{i}}{\delta-1} \text {. }
$$

Subsequently, by simple calculation, we have

$$
\begin{aligned}
E\left(D_{i}-C_{i}\right)^{2} I_{\left\{D_{i}>C_{i}\right\}} & =E\left(E\left(\left(D_{i}-c_{i}\right)^{2} I_{\left\{D_{i}>c_{i}\right\}} \mid C_{i}=c_{i}\right)\right) \\
& =E \sum_{n=c_{i}+1}^{+\infty}\left(n-c_{i}\right)^{2} \frac{1}{\delta}\left(1-\frac{1}{\delta}\right)^{n-1}=E \sum_{x=1}^{+\infty} x^{2} \frac{1}{\delta}\left(1-\frac{1}{\delta}\right)^{x+c_{i}-1} \\
& =\frac{1}{\delta} \sum_{x=1}^{+\infty} x^{2}\left(1-\frac{1}{\delta}\right)^{x-1} E\left(1-\frac{1}{\delta}\right)^{c_{i}} \\
& =\mathrm{ED}^{2} \prod_{h \in Z_{i}} y_{h}=\left(2 \delta^{2}-\delta\right) \prod_{h \in Z_{i}} y_{h},
\end{aligned}
$$

and then, by (11) and (13), we can define

$$
\begin{aligned}
& M_{1}(\pi)=\sum_{i=1}^{n}\left(\tau_{i}-\varphi_{i}\right) \prod_{h \in Z_{i}} y_{h}, \\
& M_{2}(\pi)=\left(2 \delta^{2}-\delta\right) \sum_{i=1}^{n}\left(\alpha_{i}-\beta_{i}\right) \prod_{h \in Z_{i}} y_{h} .
\end{aligned}
$$

Hence, according to (14a) and (14b), the objective in (3) and (4) can, respectively, be rewritten by

$$
\operatorname{ETP}_{1}(\pi)=M_{1}(\pi)
$$

and

$$
\operatorname{ETP}_{2}(\pi)=M_{2}(\pi)
$$

Following expectation and variance of $\left\{P_{i}\right\}$, we have

$$
\begin{aligned}
\mathrm{EC}_{i}^{2} & =E\left(\sum_{h \in Z_{i}} P_{h}^{2}+\sum_{h \in Z_{i}, h \neq l} P_{h} P_{l}\right)=\sum_{h \in Z_{i}} \mathrm{EP}_{h}^{2}+\sum_{h \in Z_{i}, h \neq l} \mathrm{EP}_{h} \mathrm{EP}_{l} \\
& =\sum_{h \in Z_{i}} \mathrm{EP}_{h}^{2}+\left(\sum_{h \in Z_{i}} \mathrm{EP}_{h}\right)^{2}-\sum_{h \in Z_{i}}\left(E P_{h}\right)^{2}=\sum_{h \in Z_{i}} \operatorname{Var} P_{h}+\left(\sum_{h \in Z_{i}} \mathrm{EP}_{h}\right)^{2} \\
& =\sum_{h \in Z_{i}} \sigma_{h}^{2}+\left(\sum_{h \in Z_{i}} \theta_{h}\right)^{2},
\end{aligned}
$$


and by (9), (17), and $\left\{P_{i}\right\}$ being independent of $\left\{D_{i}\right\}$, the objective in (5) can be further written as

$$
\begin{aligned}
\operatorname{ETP}_{3}(\pi) & =\sum_{i=1}^{n} \beta_{i}\left(\mathrm{EC}_{i}^{2}-2 \mathrm{ED}_{i} \mathrm{EC}_{i}+\mathrm{ED}_{i}^{2}\right)_{i} \\
& =\sum_{i=1}^{n} \beta_{i} \sum_{h \in Z_{i}} \sigma_{h}^{2}+\sum_{i=1}^{n} \beta_{i}\left(\sum_{h \in Z_{i}} \theta_{h}\right)^{2}-2 \delta \sum_{i=1}^{n} \beta_{i} \sum_{h \in Z_{i}} \theta_{h}+\left(2 \delta^{2}-\delta\right) \sum_{i=1}^{n} \beta_{i} \\
& =M_{3}(\pi)+M_{4}(\pi)-2 \delta M_{5}(\pi)+\left(2 \delta^{2}-\delta\right) \sum_{i=1}^{n} \beta_{i},
\end{aligned}
$$

where $M_{i}(\pi), i=3,4,5$, are similarly defined by

$$
\begin{aligned}
& M_{3}(\pi)=\sum_{i=1}^{n} \beta_{i} \sum_{h \in Z_{i}} \sigma_{h}^{2}, \\
& M_{4}(\pi)=\sum_{i=1}^{n} \beta_{i}\left(\sum_{h \in Z_{i}} \theta_{h}\right)^{2}, \\
& M_{5}(\pi)=\sum_{i=1}^{n} \beta_{i} \sum_{h \in Z_{i}} \theta_{h} .
\end{aligned}
$$

Thus, due to (15), (16), and $\operatorname{Var} P_{i}=\sigma_{i}^{2}=\theta_{i}^{2}-\theta_{i}$, (2) can be rewritten by

$$
\begin{aligned}
\operatorname{ETP}(\pi)= & \sum_{i=1}^{n}\left(\tau_{i}-\varphi_{i}\right) \prod_{h \in Z_{i}} y_{h}+\left(2 \delta^{2}-\delta\right) \sum_{i=1}^{n}\left(\alpha_{i}-\beta_{i}\right) \prod_{h \in Z_{i}} y_{h}+\sum_{i=1}^{n} \beta_{i} \sum_{h \in Z_{i}} \theta_{h}^{2} \\
& +\sum_{i=1}^{n} \beta_{i}\left(\sum_{h \in Z_{i}} \theta_{h}\right)^{2}-(2 \delta+1) \sum_{i=1}^{n} \beta_{i} \sum_{h \in Z_{i}} \theta_{h}+\left(2 \delta^{2}-\delta\right) \sum_{i=1}^{n} \beta_{i}+\sum_{i=1}^{n} \varphi_{i} .
\end{aligned}
$$

Let $Z^{*}=Z_{i}(\pi)-\{i\}=Z_{j}(\pi \prime)-\{j\}$ denote the set of jobs scheduled before job $i$ in $\pi$ (or before job $j$ in $\pi^{\prime}$ ).

Therefore, by (12) and (14a), under $\pi$ and $\pi^{\prime}$, we have

$$
\begin{aligned}
M_{1}(\pi)-M_{1}\left(\pi^{\prime}\right)= & \left(\tau_{i}-\varphi_{i}\right) y_{i} \prod_{h \in Z^{*}} y_{h}+\left(\tau_{j}-\varphi_{j}\right) y_{i} y_{j} \prod_{h \in Z^{*}} y_{h} \\
& -\left(\tau_{j}-\varphi_{j}\right) y_{j} \prod_{h \in Z^{*}} y_{h}-\left(\tau_{i}-\varphi_{i}\right) y_{j} y_{i} \prod_{h \in Z^{*}} y_{h} \\
= & \left(\left(\tau_{i}-\varphi_{i}\right) y_{i}\left(1-y_{j}\right)-\left(\tau_{j}-\varphi_{j}\right) y_{j}\left(1-y_{i}\right)\right) \prod_{h \in Z^{*}} y_{h} \\
= & \left(\left(\tau_{i}-\varphi_{i}\right) y_{i} \frac{\theta_{j} y_{j}}{\delta-1}-\left(\tau_{j}-\varphi_{j}\right) y_{j} \frac{\theta_{i} y_{i}}{\delta-1}\right) \prod_{h \in Z^{*}} y_{h} \\
= & \left(\left(\varphi_{j} \theta_{i}-\varphi_{i} \theta_{j}\right)-\left(\tau_{j} \theta_{i}-\tau_{i} \theta_{j}\right)\right) \frac{y_{i} y_{j}}{\delta-1} \prod_{h \in Z^{*}} y_{h},
\end{aligned}
$$


and according to (12), (14b), and similar process with (21),

$$
\begin{aligned}
M_{2}(\pi)-M_{2}\left(\pi^{\prime}\right)= & \left(2 \delta^{2}-\delta\right)\left[\left(\alpha_{i}-\beta_{i}\right) y_{i} \prod_{h \in Z^{*}} y_{h}+\left(\alpha_{j}-\beta_{j}\right) y_{i} y_{j} \prod_{h \in Z^{*}} y_{h}\right. \\
& \left.-\left(\alpha_{j}-\beta_{j}\right) y_{j} \prod_{h \in Z^{*}} y_{h}-\left(\alpha_{i}-\beta_{i}\right) y_{j} y_{i} \prod_{h \in Z^{*}} y_{h}\right] \\
= & \left(2 \delta^{2}-\delta\right)\left(\left(\beta_{j} \theta_{i}-\beta_{i} \theta_{j}\right)-\left(\alpha_{j} \theta_{i}-\alpha_{i} \theta_{j}\right)\right) \frac{y_{i} y_{j}}{\delta-1} \prod_{h \in Z^{*}} y_{h} .
\end{aligned}
$$

Substituting (21) into (15), (6) can be obtained, and substituting (22) into (16), (7) can be obtained.
Similarly, by (19a) and (19b),

$$
\begin{aligned}
M_{3}(\pi)-M_{3}\left(\pi^{\prime}\right)= & \beta_{i}\left(\sum_{h \in Z^{*}} \sigma_{h}^{2}+\sigma_{i}^{2}\right)+\beta_{j}\left(\sum_{h \in Z^{*}} \sigma_{h}^{2}+\sigma_{i}^{2}+\sigma_{j}^{2}\right) \\
& -\beta_{j}\left(\sum_{h \in Z^{*}} \sigma_{h}^{2}+\sigma_{j}^{2}\right)-\beta_{i}\left(\sum_{h \in Z^{*}} \sigma_{h}^{2}+\sigma_{j}^{2}+\sigma_{i}^{2}\right) \\
= & \beta_{j} \sigma_{i}^{2}-\beta_{i} \sigma_{j}^{2}=\beta_{j} \theta_{i}^{2}-\beta_{i} \theta_{j}^{2}+\beta_{i} \theta_{j}-\beta_{j} \theta_{i}, \\
M_{4}(\pi)-M_{4}\left(\pi^{\prime}\right)= & \beta_{i}\left(\sum_{h \in Z^{*}} \theta_{h}+\theta_{i}\right)^{2}+\beta_{j}\left(\sum_{h \in Z^{*}} \theta_{h}+\theta_{i}+\theta_{j}\right)^{2} \\
& -\beta_{j}\left(\sum_{h \in Z^{*}} \theta_{h}+\theta_{j}\right)^{2}-\beta_{i}\left(\sum_{h \in Z^{*}} \theta_{h}+\theta_{j}+\theta_{i}\right)^{2} \\
= & \beta_{j} \theta_{i}\left(2 \sum_{h \in Z^{*}} \theta_{h}+2 \theta_{j}+\theta_{i}-\beta_{i} \theta_{j}\left(2 \sum_{h \in Z^{*}} \theta_{h}+2 \theta_{i}+\theta_{j}\right)\right. \\
= & \left(\beta_{j} \theta_{i}-\beta_{i} \theta_{j}\right)\left(2 \sum_{h \in Z^{*}} \theta_{h}+2 \theta_{i}+2 \theta_{j}\right)+\beta_{i} \theta_{j}^{2}-\beta_{j} \theta_{i}^{2},
\end{aligned}
$$

and

$$
\begin{aligned}
M_{5}(\pi)-M_{5}\left(\pi^{\prime}\right)= & \beta_{i}\left(\sum_{h \in Z^{*}} \theta_{h}+\theta_{i}\right)+\beta_{j}\left(\sum_{h \in Z^{*}} \theta_{h}+\theta_{i}+\theta_{j}\right) \\
& -\beta_{j}\left(\sum_{h \in Z^{*}} \theta_{h}+\theta_{j}\right)-\beta_{i}\left(\sum_{h \in Z^{*}} \theta_{h}+\theta_{j}+\theta_{i}\right) \\
= & \beta_{j} \theta_{i}-\beta_{i} \theta_{j} .
\end{aligned}
$$

Substituting (23), (24), and (25) into (18), (8) can be obtained.
According to Lemma 1, when both the processing times $\left\{P_{i}\right\}$ and the due dates $\left\{D_{i}\right\}$ are geometrically distributed, we 
have the main result on the expected penalties of quadratic earliness, quadratic tardiness, and early and tardy jobs below.

Theorem 1. Assume that the processing times $P_{1}, \ldots, P_{n}$ are stochastically independent and geometrically distributed random variables with means $\theta_{1}, \ldots, \theta_{n}$, individually, and the due dates $D_{1}, \ldots, D_{n}$ follow geometric distributions with a common mean $\delta$, independent of $\left\{P_{i}\right\}$. Define

$$
\left[T_{i j}\left(1-R_{i j}\right)-y_{k} T_{j k}\left(1-R_{j k}\right)+\left(2 \delta^{2}-\delta\right)\left(\left(1-S_{i j}\right)-y_{k}\left(1-S_{j k}\right)\right)\right]<2 \theta_{k}(\delta-1)
$$

for all different, $i, j, k \in\{1,2, \ldots, n\}$,

and the jobs are firstly arranged such that in nonincreasing order of $\left\{\left(\theta_{i} / \beta_{i}\right), i=1,2, \ldots, j\right\}$, then they are arranged such that, in nondecreasing order of $\left\{\left(\theta_{i} / \beta_{i}\right), i=j, j+1, \ldots, n\right\}$, for $\exists j \in\{1,2, \ldots, n\}$, the schedule minimizes $\operatorname{ETP}(\pi)$. In other

$$
\begin{aligned}
R_{i j} & =\left(\frac{\varphi_{j}}{\theta_{j}}-\frac{\varphi_{i}}{\theta_{i}}\right)^{-1}\left(\frac{\tau_{j}}{\theta_{j}}-\frac{\tau_{i}}{\theta_{i}}\right), \\
S_{i j} & =\left(\frac{\beta_{j}}{\theta_{j}}-\frac{\beta_{i}}{\theta_{i}}\right)^{-1}\left(\frac{\alpha_{j}}{\theta_{j}}-\frac{\alpha_{i}}{\theta_{i}}\right), \\
T_{i j} & =\left(\frac{\beta_{j}}{\theta_{j}}-\frac{\beta_{i}}{\theta_{i}}\right)^{-1}\left(\frac{\varphi_{j}}{\theta_{j}}-\frac{\varphi_{i}}{\theta_{i}}\right),
\end{aligned}
$$

where $R_{i}, S_{i j}$, and $T_{i j}$ are defined unless $\varphi_{j} \theta_{i}=\varphi_{i} \theta_{j}$ or $\beta_{j} \theta_{i}=\beta_{i} \theta_{j}$.If $\left\{R_{i j}\right\},\left\{S_{i j}\right\}$, and $\left\{T_{i j}\right\}$ satisfy the following condition, words, the optimal solutions given by the schedule are $V$-shaped with respect to the order of $\left\{\theta_{i} / \beta_{i}\right\}$.

Proof. By combining (6), (7), and (8) with (26a) and (26b), we obtain

$$
\begin{aligned}
\operatorname{ETP}(\pi)-\operatorname{ETP}\left(\pi^{\prime}\right)= & \sum_{i=1}^{3}\left(\operatorname{ETP}_{i}(\pi)-\operatorname{ETP}_{i}(\pi \prime)\right) \\
= & \theta_{i} \theta_{j}\left[T_{i j}\left(\frac{\beta_{j}}{\theta_{j}}-\frac{\beta_{i}}{\theta_{i}}\right)-R_{i j} T_{i j}\left(\frac{\beta_{j}}{\theta_{j}}-\frac{\beta_{i}}{\theta_{i}}\right)\right] \frac{y_{i} y_{j}}{\delta-1} \prod_{h \in Z^{*}} y_{h} \\
& +\theta_{i} \theta_{j}\left[\left(\frac{\beta_{j}}{\theta_{j}}-\frac{\beta_{i}}{\theta_{i}}\right)-S_{i j}\left(\frac{\beta_{j}}{\theta_{j}}-\frac{\beta_{i}}{\theta_{i}}\right)\right] \frac{2 \delta^{2}-\delta}{\delta-1} y_{i} y_{j} \prod_{h \in Z^{*}} y_{h} \\
& +\theta_{i} \theta_{j}\left(\frac{\beta_{j}}{\theta_{j}}-\frac{\beta_{i}}{\theta_{i}}\right)\left(2 \sum_{h \in Z^{*}} \theta_{h}+2 \theta_{i}+2 \theta_{j}-2 \delta-1\right) .
\end{aligned}
$$

Then, by extracting common factor,

$$
\begin{aligned}
\operatorname{ETP}(\pi)-\operatorname{ETP}\left(\pi^{\prime}\right)= & \theta_{i} \theta_{j}\left(\frac{\beta_{j}}{\theta_{j}}-\frac{\beta_{i}}{\theta_{i}}\right) \times\left[\sum_{h \in Z^{*}} 2 \theta_{h}+2 \theta_{i}+2 \theta_{j}+T_{i j}\left(1-R_{i j}\right) \frac{y_{i} y_{j}}{\delta-1} \prod_{h \in Z^{*}} y_{h}\right. \\
& \left.+\left(2 \delta^{2}-\delta\right)\left(1-S_{i j}\right) \frac{y_{i} y_{j}}{\delta-1} \prod_{h \in Z^{*}} y_{h}-2 \delta-1\right] .
\end{aligned}
$$


Now, given a sequence $\pi=(\ldots, i, j, k, \ldots)$, then we denote the sequence $\pi \prime=(\ldots, j, i, k, \ldots)$ which interchanges the order of job $i$ and job $j$ in $\pi$ and the sequence $\pi^{\prime \prime}=(\ldots, i, k, j, \ldots)$ which interchanges the order of job $j$ and job $k$ in $\pi$. Define

$$
\begin{aligned}
G_{j}= & 2 \sum_{h \in Z^{*}} \theta_{h}+2 \theta_{j-1}+2 \theta_{j}+T_{j-1, j}\left(1-R_{j-1, j}\right) \frac{y_{j-1} y_{j}}{\delta-1} \prod_{h \in Z^{*}} y_{h} \\
& +\left(1-S_{j-1, j}\right) \frac{2 \delta^{2}-\delta}{\delta-1} y_{j-1} y_{j} \prod_{h \in Z^{*}} y_{h}, \\
H= & -2 \delta-1,
\end{aligned}
$$

for all $i, j, k \in\{1,2, \ldots, n\}$, and then, we obtain

$$
\operatorname{ETP}(\pi)-\operatorname{ETP}(\pi \prime)=\theta_{i} \theta_{j}\left(\frac{\beta_{j}}{\theta_{j}}-\frac{\beta_{i}}{\theta_{i}}\right)\left(G_{j}+H\right)
$$

and

$$
\operatorname{ETP}(\pi)-\operatorname{ETP}\left(\pi^{\prime \prime}\right)=\theta_{j} \theta_{k}\left(\frac{\beta_{k}}{\theta_{k}}-\frac{\beta_{j}}{\theta_{j}}\right)\left(G_{k}+H\right) .
$$

If $G_{j}+H<0$ and according to the assumption of Theorem 1, the jobs are firstly handled such that

$$
\frac{\theta_{j}}{\beta_{j}} \geq \frac{\theta_{j+1}}{\beta_{j+1}} \Leftrightarrow \frac{\beta_{i}}{\theta_{i}} \leq \frac{\beta_{j}}{\theta_{j}}
$$

Then, (31) implies

$\operatorname{ETP}(\pi)-\operatorname{ETP}(\pi \prime)=\theta_{i} \theta_{j}\left(\frac{\beta_{j}}{\theta_{j}}-\frac{\beta_{i}}{\theta_{i}}\right)\left(G_{j}+H\right) \leq 0$.

If $G_{j}+H \geq 0$ and combining (27) and (30), we deduce that

$$
\begin{aligned}
G_{k}-G_{j}= & 2 \theta_{k}+y_{k} T_{j k}\left(1-R_{j k}\right) \frac{y_{i} y_{j}}{\delta-1} \prod_{h \in Z^{*}} y_{h}+y_{k}\left(2 \delta^{2}-\delta\right)\left(1-S_{j k}\right) \frac{y_{i} y_{j}}{\delta-1} \prod_{h \in Z^{*}} y_{h} \\
& -T_{i j}\left(1-R_{i j}\right) \frac{y_{i} y_{j}}{\delta-1} \prod_{h \in Z^{*}} y_{h}-\left(2 \delta^{2}-\delta\right)\left(1-S_{i j}\right) \frac{y_{i} y_{j}}{\delta-1} \prod_{h \in Z^{*}} y_{h} \\
= & 2 \theta_{k}\left(1-\frac{q}{2 \theta_{k}(\delta-1)} y_{i} y_{j} \prod_{h \in Z^{*}} y_{h}\right)>0,
\end{aligned}
$$

where $\quad q=T_{i j}\left(1-R_{i j}\right)-y_{k} T_{j k}\left(1-R_{j k}\right)+\left(2 \delta^{2}-\delta\right)((1-$ $\left.\left.S_{i j}\right)-y_{k}\left(1-S_{j k}\right)\right)$. Hence, we obtain $G_{k}-H>G_{j}-H \geq 0$. Due to the assumption of Theorem 1 , the jobs are secondly handled such that

$$
\frac{\theta_{j+1}}{\beta_{j+1}} \geq \frac{\theta_{j}}{\beta_{j}} \Longleftrightarrow \frac{\beta_{k}}{\theta_{k}} \leq \frac{\beta_{j}}{\theta_{j}} .
$$

Then, (32) implies

$$
\operatorname{ETP}(\pi)-\operatorname{ETP}\left(\pi^{\prime \prime}\right)=\theta_{j} \theta_{k}\left(\frac{\beta_{k}}{\theta_{k}}-\frac{\beta_{j}}{\theta_{j}}\right)\left(G_{k}+H\right) \leq 0 .
$$

Thus, the result of Theorem 1 is proved that an optimal sequence to minimize $\operatorname{ETP}(\pi)$ is $\mathrm{V}$-shaped with respect to $\left\{\theta_{i} / \beta_{i}\right\}$.

For distinct $\alpha_{i}, \beta_{i}, \tau_{i}$, and $\varphi_{i}$, a large quantity of cases are covered by Theorem 1 . When the four weights are some constants, we discuss a special case below.
A special case: assume that $R_{i j} \equiv R, S_{i j} \equiv S$, and $T_{i j} \equiv T$ are some constants, then condition (27) is rewritten by

$$
T(1-R)\left(1-y_{k}\right)+\left(2 \delta^{2}-\delta\right)(1-S)\left(1-y_{k}\right)<2 \theta_{k}(\delta-1) \text {. }
$$

If $R>1, S>1$, and $T>0$, the above condition evidently holds. Therefore, $\left\{\theta_{i} / \alpha_{i}\right\},\left\{\theta_{i} / \beta_{i}\right\},\left\{\theta_{i} / \tau_{i}\right\}$, and $\left\{\theta_{i} / \varphi_{i}\right\}$ have the same order. Hence, by Theorem 1 , the optimal schedule is $\mathrm{V}$-shaped with respect to $\left\{\theta_{i} / \alpha_{i}\right\},\left\{\theta_{i} / \beta_{i}\right\},\left\{\theta_{i} / \tau_{i}\right\}$, or $\left\{\theta_{i} / \varphi_{i}\right\}$.

In addition, we, under different assumptions, show two theorems related to this $\operatorname{ETP}(\pi)$ problem where the optimal solutions exist.

Theorem 2. Assume that $\min _{1 \leq i \leq n} \theta_{i}>\delta$, and $R_{i j} \equiv R$, $S_{i j} \equiv S$, and $T_{i j} \equiv T$ are some constants.

(i) If $0<R<1,0<S<1$, and $T>0$, that is, $\left\{\theta_{i} / \alpha_{i}\right\}$, $\left\{\theta_{i} / \beta_{i}\right\},\left\{\theta_{i} / \tau_{i}\right\}$, and $\left\{\theta_{i} / \varphi_{i}\right\}$ have same orders, then 
$\operatorname{ETP}(\pi)$ is minimized by the schedule in nondecreasing order of $\left\{\theta_{i} / \alpha_{i}\right\},\left\{\theta_{i} / \beta_{i}\right\},\left\{\theta_{i} / \tau_{i}\right\}$, or $\left\{\theta_{i} / \varphi_{i}\right\}$

(ii) If $R<0, S<0$, and $T>0$, that is, $\left\{\theta_{i} / \beta_{i}\right\}$ and $\left\{\theta_{i} / \varphi_{i}\right\}$ have the same orders, $\left\{\theta_{i} / \alpha_{i}\right\}$ and $\left\{\theta_{i} / \tau_{i}\right\}$ have the same orders, and $\left\{\theta_{i} / \beta_{i}\right\}$ and $\left\{\theta_{i} / \alpha_{i}\right\}$ have opposite orders, then $\operatorname{ETP}(\pi)$ is minimized by the schedule in nondecreasing order of $\left\{\theta_{i} / \beta_{i}\right\}$ or $\left\{\theta_{i} / \varphi_{i}\right\}$, or, equivalently, in nonincreasing order of $\left\{\theta_{i} / \alpha_{i}\right\}$ or $\left\{\theta_{i} / \tau_{i}\right\}$

(iii) If $R>1, S<0$ and $T<0$, that is, $\left\{\theta_{i} / \alpha_{i}\right\},\left\{\theta_{i} / \tau_{i}\right\}$, and $\left\{\theta_{i} / \varphi_{i}\right\}$ have same orders and $\left\{\theta_{i} / \beta_{i}\right\}$ and $\left\{\theta_{i} / \alpha_{i}\right\}$ have opposite orders, then $\operatorname{ETP}(\pi)$ is minimized by the schedule in nondecreasing order of $\left\{\theta_{i} / \beta_{i}\right\}$, or, equivalently, in nonincreasing order of $\left\{\theta_{i} / \alpha_{i}\right\}$, $\left\{\theta_{i} / \tau_{i}\right\}$, or $\left\{\theta_{i} / \varphi_{i}\right\}$

Theorem 3. Assume that $\sum_{i=1}^{n} \theta_{i}<\delta$, and $R_{i j} \equiv R, S_{i j} \equiv S$, and $T_{i j} \equiv T$ are some constants.

(i) If $R>1, S>1$, and $T>0$, that is, $\left\{\theta_{i} / \alpha_{i}\right\},\left\{\theta_{i} / \beta_{i}\right\}$, $\left\{\theta_{i} / \tau_{i}\right\}$, and $\left\{\theta_{i} / \varphi_{i}\right\}$ have the same orders, then $\operatorname{ETP}(\pi)$ is minimized by the schedule in nonincreasing order of $\left\{\theta_{i} / \alpha_{i}\right\},\left\{\theta_{i} / \beta_{i}\right\},\left\{\theta_{i} / \tau_{i}\right\}$, or $\left\{\theta_{i} / \varphi_{i}\right\}$

(ii) If $0<R<1, S>1$, and $T<0$, that is, $\left\{\theta_{i} / \beta_{i}\right\}$ and $\left\{\theta_{i} / \alpha_{i}\right\}$ have the same orders, $\left\{\theta_{i} / \tau_{i}\right\}$ and $\left\{\theta_{i} / \varphi_{i}\right\}$ have the same orders, and $\left\{\theta_{i} / \beta_{i}\right\}$ and $\left\{\theta_{i} / \varphi_{i}\right\}$ have opposite orders, then $\operatorname{ETP}(\pi)$ is minimized by the schedule in nonincreasing order of $\left\{\theta_{i} / \beta_{i}\right\}$ or $\left\{\theta_{i} / \alpha_{i}\right\}$, or, equivalently, in nondecreasing order of $\left\{\theta_{i} / \tau_{i}\right\}$ or $\left\{\theta_{i} / \varphi_{i}\right\}$

(iii) If $R<0, S>1$, and $T<0$, that is, $\left\{\theta_{i} / \beta_{i}\right\},\left\{\theta_{i} / \alpha_{i}\right\}$, and $\left\{\theta_{i} / \tau_{i}\right\}$ have the same orders and $\left\{\theta_{i} / \beta_{i}\right\}$ and $\left\{\theta_{i} / \varphi_{i}\right\}$ have opposite orders, then $\operatorname{ETP}(\pi)$ is minimized by the schedule in nonincreasing order of $\left\{\theta_{i} / \beta_{i}\right\},\left\{\theta_{i} / \alpha_{i}\right\}$, or $\left\{\theta_{i} / \tau_{i}\right\}$, or, equivalently, in nondecreasing order of $\left\{\theta_{i} / \varphi_{i}\right\}$

Proof. Let $\left(\theta_{i} / \alpha_{i}\right) \leq\left(\theta_{j} / \alpha_{j}\right),\left(\theta_{i} / \beta_{i}\right) \leq\left(\theta_{j} / \beta_{j}\right),\left(\theta_{i} / \tau_{i}\right) \leq\left(\theta_{j} /\right.$ $\left.\tau_{j}\right)$, and $\left(\theta_{i} / \varphi_{i}\right) \leq\left(\theta_{j} / \varphi_{j}\right)$; according to the assumption that $\min _{1 \leq i \leq n} \theta_{i}>\delta$ and the conditions of Part (i), we have $T_{i j}(1-$ $\left.R_{i j}\right)>0$ and $1-S_{i j}>0$. It follows from (29) that ETP $(\pi)-\operatorname{ETP}(\pi \prime) \leq 0$. This proves that $\operatorname{ETP}(\pi)-\operatorname{ETP}(\pi \prime) \leq 0$ if and only if $\left(\theta_{i} / \alpha_{i}\right) \leq\left(\theta_{j} / \alpha_{j}\right),\left(\theta_{i} / \beta_{i}\right) \leq\left(\theta_{j} / \beta_{j}\right),\left(\theta_{i} / \tau_{i}\right) \leq$ $\left(\theta_{j} / \tau_{j}\right)$, or $\left(\theta_{i} / \varphi_{i}\right) \leq\left(\theta_{j} / \varphi_{j}\right)$.

According to the similar proof procedure of Part (i), we can prove Part (ii), Part (iii), Part (iv), Part (v), and Part (vi).

\section{Dynamic Programming Algorithm}

In this section, in order to obtain the optimal solution of this stochastic problem $\operatorname{ETP}(\pi)$ among all V-shaped schedules, we propose dynamic programming algorithm based on Theorem 1 for the objective function (20). In general, it is necessary for the jobs to be numbered in nondecreasing order of $\left\{\theta_{i} / \beta_{i}\right\}$, i.e., $\left(\theta_{1} / \beta_{1}\right) \leq\left(\theta_{2} / \beta_{2}\right) \leq \ldots \leq\left(\theta_{n} / \beta_{n}\right)$. Then, think about a set of the jobs $A_{i}=\{1,2, \ldots, i\}$ for $i \in\{1,2, \ldots, n\}$. Hence, job can be scheduled as the first or the last in this schedule for all the jobs in $A_{i}$ if a schedule has a V-shaped characteristic. Suppose that $\pi^{*}$ is denoted as the optimal V-shaped schedule for the objective function (20) and $A_{i}^{\prime}$ represents a set of the jobs is scheduled before those jobs in $A_{i}$ under $\pi^{*}$. Let $\Psi_{i}^{\prime}=\prod_{h \in A_{i}^{\prime}} y_{h}, \Upsilon_{i}^{\prime}=\sum_{h \in A_{i}^{\prime}} \theta_{h}$, and $\Omega_{i}^{\prime}=\sum_{h \in A_{i}^{\prime}} \theta_{h}^{2}$. Then, provided $\Psi_{i}^{\prime}, \Upsilon_{i}^{\prime}$, and $\Omega_{i}^{\prime}, f_{i}\left(\Psi_{i}^{\prime}, \Upsilon_{i}^{\prime}, \Omega_{i}^{\prime}\right)$ is defined as the contribution of all the jobs in $A_{i}$ to the objective function (20). Afterwards, it is obvious that the contribution of the jobs if job $i$ is the first and last job scheduled among all jobs in $A_{i}$ is, respectively,

$$
\begin{aligned}
f_{i}^{a}\left(\Psi_{i}^{\prime}, \Upsilon_{i}^{\prime}, \Omega_{i}^{\prime}\right)= & f_{i-1}\left(\Psi_{i}^{\prime} y_{i}, \Upsilon_{i}^{\prime}+\theta_{i}, \Omega_{i}^{\prime}+\theta_{i}^{2}\right)+\left(\left(\tau_{i}-\varphi_{i}\right)+\left(2 \delta^{2}-\delta\right)\left(\alpha_{i}-\beta_{i}\right)\right) \Psi_{i}^{\prime} y_{i} \\
& +\beta_{i}\left(\left(\Omega_{i}^{\prime}+\theta_{i}^{2}\right)+\left(\Upsilon_{i}^{\prime}+\theta_{i}\right)^{2}\right)-(2 \delta+1) \beta_{i}\left(\Upsilon_{i}^{\prime}+\theta_{i}\right)
\end{aligned}
$$

and

$$
\begin{aligned}
f_{i}^{b}\left(\Psi_{i}^{\prime}, \Upsilon_{i}^{\prime}, \Omega_{i}^{\prime}\right)= & f_{i-1}\left(\Psi_{i}^{\prime}, \Upsilon_{i}^{\prime}, \Omega_{i}^{\prime}\right)+\left(\left(\tau_{i}-\varphi_{i}\right)+\left(2 \delta^{2}-\delta\right)\left(\alpha_{i}-\beta_{i}\right)\right) \Psi_{i}^{\prime} \Psi_{i} \\
& +\beta_{i}\left(\left(\Omega_{i}^{\prime}+\Omega_{i}\right)+\left(\Upsilon_{i}^{\prime}+\Upsilon_{i}\right)^{2}\right)-(2 \delta+1) \beta_{i}\left(\Upsilon_{i}^{\prime}+\Upsilon_{i}\right),
\end{aligned}
$$

where $\Psi_{i}=\prod_{h \in A_{i}} y_{h}, \Upsilon_{i}=\sum_{h \in A_{i}} \theta_{h}$, and $\Omega_{i}=\sum_{h \in A_{i}} \theta_{h}^{2}$.

On the basis of the optimal principle of dynamic programming, and $f_{i}^{a}\left(\Psi_{i}^{\prime}, \Upsilon_{i}^{\prime}, \Omega_{i}^{\prime}\right)$ and $f_{i}^{b}\left(\Psi_{i}^{\prime}, Y_{i}^{\prime}, \Omega_{i}^{\prime}\right)$ are expressed in (39a) and (39b), respectively, hence the jobs are handled by the optimal V-shaped schedule such that

$$
f_{i}\left(\Psi_{i}^{\prime}, \Upsilon_{i}^{\prime}, \Omega_{i}^{\prime}\right)=\min \left\{f_{i}^{a}\left(\Psi_{i}^{\prime}, \Upsilon_{i}^{\prime}, \Omega_{i}^{\prime}\right), f_{i}^{b}\left(\Psi_{i}^{\prime}, \Upsilon_{i}^{\prime}, \Omega_{i}^{\prime}\right)\right\}+\left(2 \delta^{2}-\delta\right) \sum_{h \in A_{i}} \beta_{i}+\sum_{h \in A_{i}} \varphi_{i}
$$


Step 1: for $i=1,2, \ldots, n$, we can calculate $f_{i}\left(\Psi_{i}^{\prime}, \Upsilon_{i}^{\prime}, \Omega_{i}^{\prime}\right)$ by (39a), (39b), and (40) for all possible values $\Psi_{i}^{\prime}, Y_{i}^{\prime}$, and $\Omega_{i}^{\prime}$. Step 2: define $F_{n}^{*}=\min _{\Psi_{t}^{\prime} Y_{n}^{\prime} \Omega_{n}^{\prime}}\left(f_{n}\left(\Psi_{n}^{\prime}, \Upsilon_{n}^{\prime}, \Omega_{n}^{\prime}\right)\right)$

Step 3: structure the optimal V-shaped schedule $\pi^{*}$ that obtains $F_{n}^{*}$ due to the backward tracking process

Algorithm 1: Dynamic programming.

Thus, we can summarize the dynamic programming algorithm designed to achieve the optimal V-shaped schedule for this stochastic JIT problem.

Since this is a dynamic programming algorithm, we can obtain $\pi^{*}$ according to its standard procedure, and the detailed process of the backward tracking is omitted. The algorithm is used to calculate all $f_{i}\left(\Psi_{i}^{\prime}, \Upsilon_{i}^{\prime}, \Omega_{i}^{\prime}\right)$, at most $\mathrm{O}\left(\Psi_{i}^{\prime} \Upsilon_{i}^{\prime} \Omega_{i}^{\prime}\right)$ steps, for each $i$. Thus, Algorithm 1 can derive the optimal schedule in $\mathrm{O}\left(n \Psi_{i}^{\prime} \Upsilon_{i}^{\prime} \Omega_{i}^{\prime}\right)$ pseudopolynomial time for the stochastic single machine JIT problem.

\section{Conclusions}

In this paper, we study a static stochastic single machine JIT scheduling problem in which the processing times are stochastically independent and geometrically distributed with distinct parameters, the due dates are geometrically distributed with a common parameter, and both the unit penalty of earliness/tardiness and the fixed penalty of earliness/tardiness are certain and distinct, where the JIT scheduling concept means that all the jobs are scheduled to complete as close to their due dates as possible. The objective is to find a schedule of jobs that minimizes the expected total penalties for quadratic earliness, quadratic tardiness, and early and tardy jobs. We show that an optimal schedule minimizing this problem has a V-shaped characteristic with respect to $\left\{\theta_{i} / \beta_{i}\right\}$ by mathematical derivation under the specific condition (27). That is, the schedule will first arrange jobs in nonincreasing order of $\left\{\theta_{i} / \beta_{i}\right\}$ and then arrange jobs in nondecreasing order of $\left\{\theta_{i} / \beta_{i}\right\}$. Nevertheless, there is a special case under three constants with specific value range, namely, $R_{i j}, S_{i j}$, and $T_{i j}$, and then, the optimal solution obtained is $\mathrm{V}$-shaped with respect to $\left\{\theta_{i} / \alpha_{i}\right\},\left\{\theta_{i} / \beta_{i}\right\},\left\{\theta_{i} / \tau_{i}\right\}$, or $\left\{\theta_{i} / \varphi_{i}\right\}$. Furthermore, we also give two theorems which contain a total of six situations related to this problem under different assumptions, and then, the optimal solution obtained is either monotonous nonincreasing or monotonous nondecreasing with respect to the ratio of mean processing time to different penalties. Finally, based on the V-shaped characteristic, we develop a dynamic programming algorithm which is designed to obtain an optimal $\mathrm{V}$-shaped schedule in pseudopolynomial time.

As a direction for the future research, it may be an interesting and significant challenge to consider this problem with other distributed processing times and due dates. In addition, this problem would be worth extending towards more complex systems such as parallel processors or dedicated processors.

\section{Data Availability}

No data were used to support the findings of the study.

\section{Conflicts of Interest}

The author declares that there are no conflicts of interest reported in this paper.

\section{References}

[1] J. B. Sidney, "Optimal single-machine scheduling with earliness and tardiness penalties," Operations Research, vol. 25, no. 1, pp. 62-69, 1977.

[2] J. J. Kanet, "Minimizing the average deviation of job completion times about a common due date," Naval Research Logistics Quarterly, vol. 28, no. 4, pp. 643-651, 1981.

[3] A. Lann and G. Mosheiov, "Single machine scheduling to minimize the number of early and tardy jobs," Computers \& Operations Research, vol. 23, no. 8, pp. 769-781, 1996.

[4] C.-F. Liaw, "A branch-and-bound algorithm for the single machine earliness and tardiness scheduling problem," Computers \& Operations Research, vol. 26, no. 7, pp. 679-693, 1999.

[5] C. M. Hino, D. P. Ronconi, and A. B. Mendes, "Minimizing earliness and tardiness penalties in a single-machine problem with a common due date," European Journal of Operational Research, vol. 160, no. 1, pp. 190-201, 2005.

[6] R. M'Hallah, "Minimizing total earliness and tardiness on a single machine using a hybrid heuristic," Computers \& Operations Research, vol. 34, no. 10, pp. 3126-3142, 2007.

[7] V. Kayvanfar, I. Mahdavi, and G. M. Komaki, "Single machine scheduling with controllable processing times to minimize total tardiness and earliness," Computers \& Industrial Engineering, vol. 65, no. 1, pp. 166-175, 2013.

[8] V. Kayvanfar, I. Mahdavi, and GH. M. Komaki, "A drastic hybrid heuristic algorithm to approach to JIT policy considering controllable processing times," International Journal of Advanced Manufacturing Technology, vol. 69, no. 1-4, pp. 257-267, 2013.

[9] Y. Hendel, N. Runge, and F. Sourd, "The one-machine just-intime scheduling problem with preemption," Discrete Optimization, vol. 6, no. 1, pp. 10-22, 2009.

[10] H. Khorshidian, N. Javadian, M. Zandieh, J. Rezaeian, and K. Rahmani, "A genetic algorithm for JIT single machine scheduling with preemption and machine idle time," Expert Systems with Applications, vol. 38, no. 7, pp. 7911-7918, 2011.

[11] S. K. Gupta and T. Sen, "Minimizing a quadratic function of job lateness on a single machine," Engineering Costs and Production Economics, vol. 7, no. 3, pp. 187-194, 1983.

[12] U. Bagchi, R. S. Sullivan, and Y.-L. Chang, "Minimizing mean squared deviation of completion times about a common due date," Management Science, vol. 33, no. 7, pp. 894-906, 1987. 
[13] J. Schaller, "Minimizing the sum of squares lateness on a single machine," European Journal of Operational Research, vol. 143, no. 1, pp. 64-79, 2002.

[14] X. Weng and J. A. Ventura, "Scheduling about a given common due date to minimize mean squared deviation of completion times," European Journal of Operational Research, vol. 88, no. 2, pp. 328-335, 1996.

[15] T. Sen, P. Dileepan, and M. R. Lind, "Minimizing a weighted quadratic function of job lateness in the single machine system," International Journal of Production Economics, vol. 42, no. 3, pp. 237-243, 1996.

[16] J. M. S. Valente and M. R. A. Moreira, "Greedy randomised dispatching heuristics for the single machine scheduling problem with quadratic earliness and tardiness penalties," International Journal of Advanced Manufacturing Technology, vol. 44, no. 9-10, pp. 995-1009, 2009.

[17] J. M. S. Valente, M. R. A. Moreira, A. Singh, and R. A. F. S. Alves, "Genetic algorithms for single machine scheduling with quadratic earliness and tardiness costs," International Journal of Advanced Manufacturing Technology, vol. 54, no. 1-4, pp. 251-265, 2011.

[18] K. Kianfar and G. Moslehi, "A branch-and-bound algorithm for single machine scheduling with quadratic earliness and tardiness penalties," Computers \& Operations Research, vol. 39, no. 12, pp. 2978-2990, 2012.

[19] J. Schaller, "Single machine scheduling with early and quadratic tardy penalties," Computers \& Industrial Engineering, vol. 46, no. 3, pp. 511-532, 2004.

[20] K. Rahmani and I. Mahdavi, "A genetic algorithm for the single machine preemptive scheduling problem with linear earliness and quadratic tardiness penalties," The International Journal of Advanced Manufacturing Technology, vol. 65, no. 58, pp. 763-770, 2013.

[21] C.-Y. Lee, S. L. Danusaputro, and C.-S. Lin, "Minimizing weighted number of tardy jobs and weighted earliness-tardiness penalties about a common due date," Computers \& Operations Research, vol. 18, no. 4, pp. 379-389, 1991.

[22] D. Shabtay, "Due date assignments and scheduling a single machine with a general earliness/tardiness cost function," Computers \& Operations Research, vol. 35, no. 5, pp. 15391545, 2008.

[23] C. Koulamas, "Common due date assignment with generalized earliness/tardiness penalties," Computers \& Industrial Engineering, vol. 109, pp. 79-83, 2017.

[24] K. R. Baker and G. D. Scudder, "Sequencing with earliness and tardiness penalties: a review," Operations Research, vol. 38, no. 1, pp. 22-36, 1990.

[25] G. A. Rolim and M. S. Nagano, "Structural properties and algorithms for earliness and tardiness scheduling against common due dates and windows: a review," Computers \& Industrial Engineering, vol. 149, Article ID 106803, 2020.

[26] Ö. Hazır and S. Kedad-Sidhoum, "Batch sizing and just-intime scheduling with common due date," Annals of Operations Research, vol. 213, no. 1, pp. 187-202, 2014.

[27] L. Kong, H. Li, H. Luo, L. Ding, X. Luo, and M. Skitmore, "Optimal single-machine batch scheduling for the manufacture, transportation and JIT assembly of precast construction with changeover costs within due dates," Automation in Construction, vol. 81, pp. 34-43, 2017.

[28] H. M. Soroush and L. D. Fredendall, "The stochastic single machine scheduling problem with earliness and tardiness costs," European Journal of Operational Research, vol. 77, no. 2, pp. 287-302, 1994.
[29] K. R. Baker, "Minimizing earliness and tardiness costs in stochastic scheduling," European Journal of Operational Research, vol. 236, no. 2, pp. 445-452, 2014.

[30] X. Q. Cai, X. Y. Wu, and X. Zhou, Optimal Stochastic Scheduling, Springer, New York, NY, USA, 2014.

[31] M. Liu and X. Liu, "Machine scheduling with stochastic release and processing times," IFAC-PapersOnLine, vol. 52, no. 13, pp. 2116-2121, 2019.

[32] X. Cai and S. Zhou, "Scheduling stochastic jobs with asymmetric earliness and tardiness penalties," Naval Research Logistics, vol. 44, no. 6, pp. 531-557, 1997.

[33] H. M. Soroush, "Minimizing the weighted number of early and tardy jobs in a stochastic single machine scheduling problem," European Journal of Operational Research, vol. 181, no. 1, pp. 266-287, 2007.

[34] V. Portougal and D. Trietsch, "Setting due dates in a stochastic single machine environment," Computers \& Operations Research, vol. 33, no. 6, pp. 1681-1694, 2006.

[35] R. Benmansour, H. Allaoui, and A. Artiba, "Stochastic single machine scheduling with random common due date," International Journal of Production Research, vol. 50, no. 13, pp. 3560-3571, 2012.

[36] R. F. Lemos and D. P. Ronconi, "Heuristics for the stochastic single-machine problem with E/T costs," International Journal of Production Economics, vol. 168, no. 10, pp. 131-142, 2015.

[37] J. Mittenthal and M. Raghavachari, "Stochastic single machine scheduling with quadratic early-tardy penalties," Operations Research, vol. 41, no. 4, pp. 786-796, 1993.

[38] X. Cai and X. Zhou, "Asymmetric earliness and tardiness scheduling with exponential processing times on an unreliable machine," Annals of Operations Research, vol. 98, no. 1/4, pp. 313-331, 2000.

[39] X. Cai, L. Wang, and X. Zhou, "Single-machine scheduling to stochastically minimize maximum lateness," Journal of Scheduling, vol. 10, no. 4-5, pp. 293-301, 2007.

[40] H. M. Soroush and F. A. Alqallaf, "Minimising a weighted quadratic function of job lateness in the stochastic single machine scheduling problem," International Journal of Operational Research, vol. 6, no. 4, pp. 538-572, 2009.

[41] H. M. Soroush, "Solving a stochastic single machine problem with initial idle time and quadratic objective," Computers \& Operations Research, vol. 37, no. 7, pp. 1328-1347, 2010. 